\title{
Human infection due to Mycobacterium marinum after a dolphin bite
}

\author{
D. J. FLOWERS
}

From the Department of Pathology, Royal Sussex County Hospital, Brighton, Sussex

SYNOPSIS A young man employed at the local aquarium was bitten by a bottlenose dolphincs (Tursiops truncatus) during a training session, receiving a slight injury which healed rapidly. $\frac{\mathbb{O}}{\mathbb{O}}$ Some two months later fluctuant swellings appeared in the region of the bite, which developed $\stackrel{\mathbb{D}}{\rightrightarrows}$ into indolent ulcers which have not completely healed seven months after the original bite. Cultures taken on two oscasions have yielded a pure growth of Mycobacterium marinum.

\section{Case Report}

The patient, R.S., a young man aged 19 years, received a bite on 30 November 1968 while training the dolphins. In order to achieve a successful trainer/dolphin relationship it is sometimes necessary to enter the pool and swim with these animals, and it was during such a session that the patient was bitten. The patient attended the Casualty Department at this hospital on the following day and was found to have a superficial wound on the right middle finger, which was dressed, and he was given tetanus toxoid and Triplopen. After two days he received a further dose of Triplopen and the wound healed uneventfully within a week. On 20 January 1969 he noticed that several swellings had appeared, which persisted, and he visited his general practitioner who found a painless swelling on the right forearm, one just proximal to the bite, and a third on the dorsum of the hand which somewhat resembled a ganglion. The lesions felt encysted and were fluctuant but there was no axillary lymphadenopathy.

He was referred to this hospital on 13 February 1969 at which time the swellings had persisted but were not increasing in size (Fig. 1) and were still painless unless they were knocked. Since they did not appear inflammatory no antibiotics were given, but one lesion was aspirated yielding approximately $1 \mathrm{ml}$ of thick, viscid pus, and a course of Received for publication 23 December 1969. ultraviolet therapy was begun. He was seen again on 20 February when the cultures taken from the specimen of aspirated pus had yielded no growth, $\mathbb{\perp}$ and the remaining lesions were aspirated and one $\stackrel{2}{\Rightarrow}$ was removed for section. When seen on 24 April 1969 the lesions were still discharging (Fig. 2) but on 22 May, apart from a small ulcer on the knuckle of the right middle finger, all the lesions had healed.

In an earlier series of cases (Linell and Nordén, 1954), where Myco. marinum was the infective agent, lesions healed spontaneously but in severalô cases this took one to two years.

\section{Investigations}

Due to the nature of the original wound the $\tilde{D}$ specimens of aspirated pus were submitted to extensive examination. Wet preparations were examined by dark-ground and phase-contrasto microscopy, and films stained by Gram and ZiehlNeelsen were thoroughly searched. Apart from $\stackrel{?}{+}$ large numbers of neutrophils nothing of note was 0 seen. This, although disappointing at the time, in retrospect is not surprising since in the series of $\cong$ Linell and Nordén (1954) acid-fast bacilli were $\stackrel{\odot}{\varnothing}$ seen in only one case out of 33 positive by culture. Enquiries made to veterinarians, the London Zoo, and various Ministry laboratories suggested the응 possibility of an erysipeloid lesion but failed to 


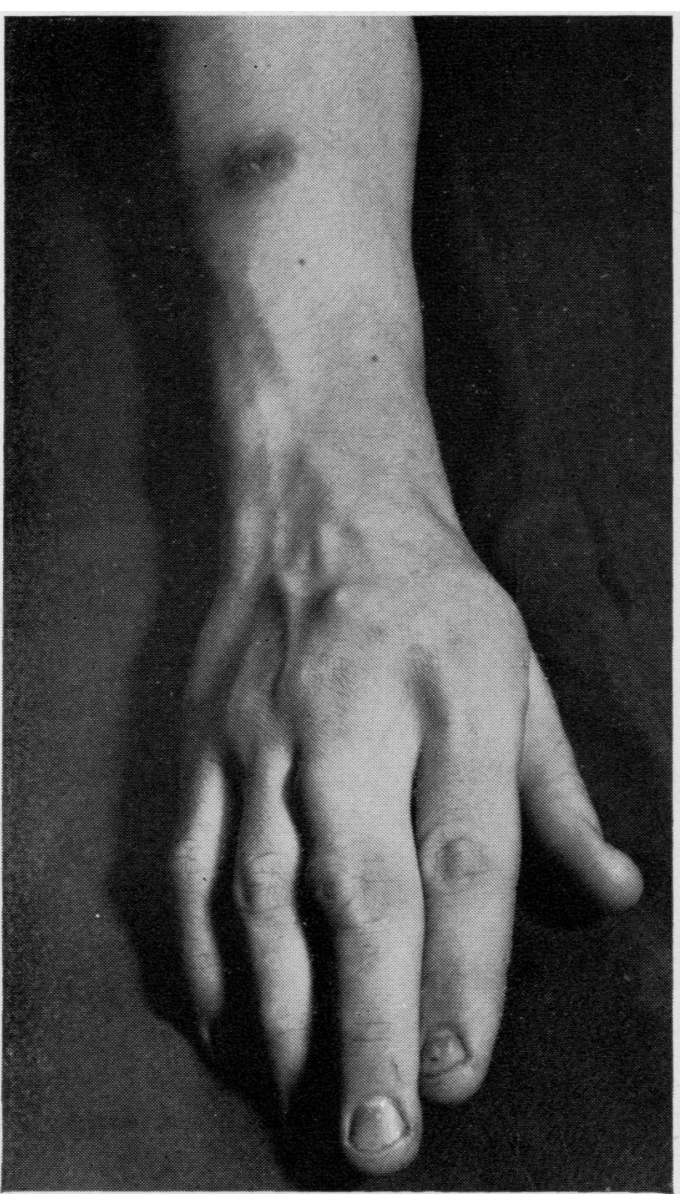

Fig. 1 The infected area when the patient was referred to hospital.

reveal similar cases or information on the flora carried by dolphins. Culture media were therefore used which would cover the possibility of infection with mycobacteria, mycoplasma, leptospira, erysipelothrix, and fungi, in addition to common pyogenic species. Each medium was inoculated in triplicate, cultures being held at room temperature, $28^{\circ} \mathrm{C}$, and $37^{\circ} \mathrm{C}$, and an additional sample was sent to the local public health laboratory for virus culture. After eight days' incubation at $28^{\circ} \mathrm{C}$ tiny pinpoint colonies were seen on the Lowenstein-Jensen slope and films of these showed acid-fast bacilli. Within the following week similar colonies appeared on Dorset egg, blood agar, and serum agar slopes held at $28^{\circ} \mathrm{C}$, and later in the same culture media held at room temperature.

No growth occurred in the cultures held at $37^{\circ} \mathrm{C}$ for one month, and no other bacteria, fungi, or viruses were isolated from any of the cultures used. Similar findings were made with the

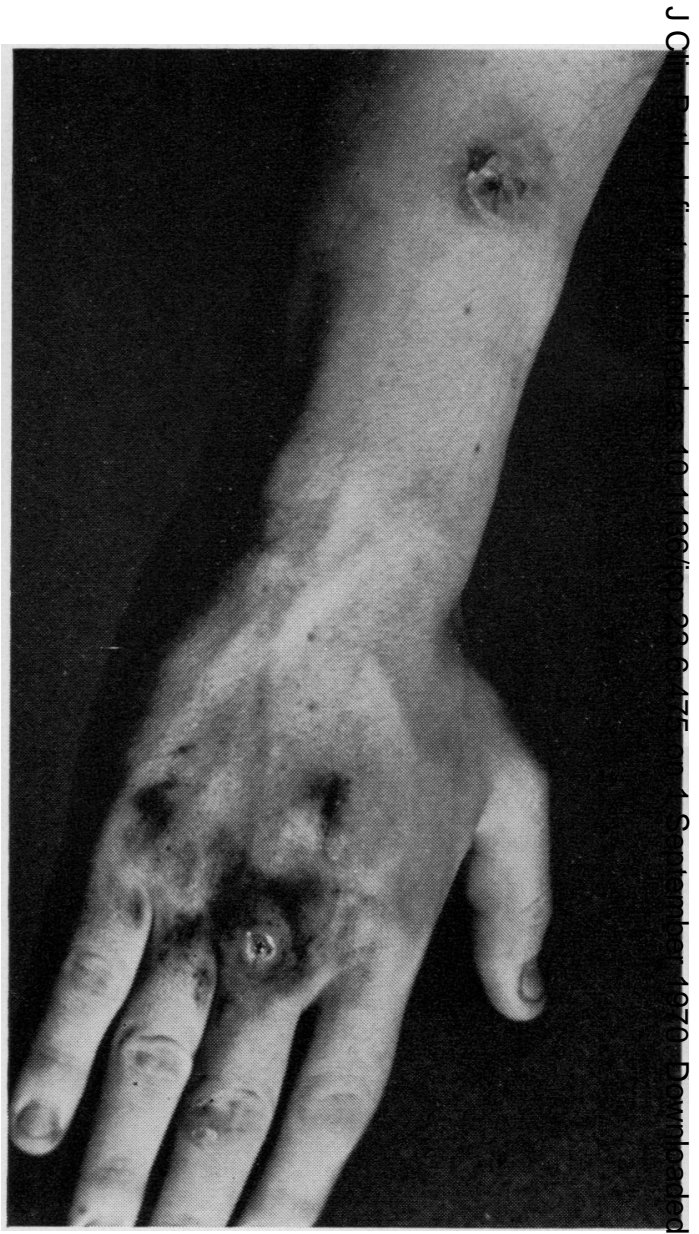

Fig. 2. The infected area two months later.

specimen aspirated on 20 February 1969.

After three weeks at $28^{\circ} \mathrm{C}$ colonies were $3-4 \mathrm{~mm}^{3}$ in diameter, rough in texture, and deep orange in colour. They were referred to $\mathrm{Mr} \mathrm{C}$. $\mathrm{H}_{\gg}$ Collins of the Tuberculosis Reference Laboratory을. Westminster, for identification and sensitivity testing. They were found to be Mycobacterium marinum (syn. Myco balnei) sensitive at $25^{\circ} \mathrm{C}$ and $33^{\circ} \mathrm{C}$ to cycloserine, ethionamide, and ethambutol but resistant to streptomycin, PAS, INAHO thiosemicarbazone, rifampicin, viomycin, kannaco mycin, and capreomycin. A culture was also sento to Dr J. Marks at the Cardiff TB Reference ${ }^{+}$ Laboratory, who confirmed the identification and found the strain to be sensitive to Septrin by the disc method. The biopsy specimen taken on 200 February 1969 showed granulation tissue of pyo $\mathbb{Q}$ genic pattern, with no demonstrable tuberculoid foci. In spite of an exhaustive examination no acid-fast bacilli were found in sections stained byo the Ziehl Neelsen method. 
Since Myco. marinum is known to occur in sea water it seemed possible that the wound had become infected after the bite and samples were taken from the dolphin pool and a second pool containing seals, the latter being used as a control. Samples were run through a membrane filter which was then placed in Kirchmer's medium and incubated at $28^{\circ} \mathrm{C}$ for one month. Acid-fast bacilli were recovered from the dolphin pool but not from the control sample. They were not, however, isolated in pure culture and a positive identification was impossible due to large numbers of contaminating species but it seems likely that they were in fact Myco. marinum. The water in the pool is continuously filtered and intermittent daily chlorination, using $5 \mathrm{ppm}$, is added being allowed to evaporate off. It thus seems likely that the infection was contracted in the pool and that the mycobacterium is specifically associated with dolphins. The animals, however, were in perfect health at the time and have shown no signs of illness since, and it would seem that if mycobacteria are being carried by them it is in a commensal rather than pathogenic role. Several samples of frozen herring used as foodstuff were also tested, since they seemed a possible source of infection, but no mycobacteria were isolated.

\section{Discussion}

Human infection due to Myco.marinum is unusual but well recognized, several reports of 'swimming bath infection' due to this species being recorded in the literature (Linell and Nordén, 1954; Schaefer and Davis, 1961). Whilst skin infections of those who handle fish and cetacean mammals are well recognized in industry, being given names such as 'speck finger', 'sealers' finger', 'blubber finger' (Skinner, 1957; Jellison, 1951), these are invariably due to Erysipelothrix indiosa which is well recognized as a pathogen in medical and veterinary practice. The present case is of interest in associating human mycobacterial infection with an aquatic mammal, since, while studies of the dolphin's bacterial flora have been made (Ridgway, 1965), there is no evidence of mycobacterial carriage or infection in this animal. Other pinniped mammals, such as seals and sea lions, are known to harbour mycobacteria although the species involved has not been determined. We have in this group had a patient who contracted pulmonary tuberculosis following the bite of a tuberculous seal; this patient was diagnosed on lung radiological and clinical findings, but cultures for tuberculosis were consistently negative having been incubated at $37^{\circ} \mathrm{C}$ only.

The dolphin is a member of the order Cetacea which includes whales and porpoises. The terms $\frac{\mathcal{C}}{\overline{5}}$ 'dolphin' and 'porpoise' are variously used in 0 different parts of the world, often for a single $\stackrel{0}{F}$ species but the former is usually used to indicate $\underline{0}$ those cetaceans which have an elongated beak whereas in the porpoise the mouth is merely an $\stackrel{5}{9}$ opening in the rather blunt-shaped head (Harrison and King, 1965). The dolphin has long been $\frac{\overline{ }}{\bar{N}}$ regarded as a friend of man, and accounts from $\frac{\bar{p}}{7}$ modern times as far back as Greek and Roman $\stackrel{\varnothing}{\varnothing}$ mythology record cases of dolphins allowing $ळ$ children to ride on them, aiding the drowning, $\vec{P}$ piloting ships, assisting in the netting of fish, etc, $?$ an excellent account of the subject being given by $\vec{\omega}$ Alpers (1960). This natural rapport with man is $\stackrel{S}{\Omega}$ unique in an undomesticated animal and facilitates exploitation of the other unusual features which if it possesses. The high brain weight/body weight o ratio of this well cerebrated animal places it high in the phylogenetic scale with an intelligence or which, according to some workers, is second only 을 to that of man. This intelligence coupled with a vocal range in air and water possibly gives the $\mathcal{D}$ dolphins the power of communication with man (Lilly, 1962), a power of use in national defence, $\frac{\Phi}{9}$ since the sounds emitted are normally used for the detection of underwater objects on a sonar or echo location principle. However, the greatest exploit- $\vec{\theta}$ ation of this animal has been in the field of $O$ entertainment where its great agility, with speeds of 20 to 30 knots and 20-foot leaps into the air, attracts the large crowds which ensure economic viability. The increasing use of the dolphin in scientific research and entertainment will increase $\mathbb{D}$ the possibility of zoonotic infection, and more knowledge of the commensal and pathogenic flora of this animal will be needed in order to evaluate the risks involved.

I wish to thank Professor R. J. Harrison and Dr R. I. K. Elliott for assistance in the preparation of $\delta$ this paper, and $\mathrm{Mr} \mathrm{W}$. Austin-Brown for permission to publish the case history. I am also 음 indebted to Mr S. T. Higgins for the photographs.

\section{References}

Alpers, A. (1960). A Book of Dolphins, Murray, London.

Harrison, R. J., and King, J. E. (1965), Marine Mammals. Hutchinson London.

Jellison, W. L. (1951). Report of Rocky Mountains Laboratory. Federal Security Agency, Hamilton, Montana, USA.

Lilly, J. C. (1962). 'Man and Dolphin', Gollancz, London.
Linell, F., and Nordén, A. (1954). Mycobacterium balnei. A new acid fast bacillus occurring in swimming pools and capable $\mathscr{D}$ of producing skin lesions in humans. Acta tuberc. scand., Suppl 33.

Ridgway, S. H. (1965). Medical care of marine mammals, J.Am.vet. med. Ass., 147, 1077-1085.

Schaefer, W. B., and Davis, C. L. (1961). A bacteriologic and histopathologic study of skin granuloma due to $\mathrm{Myco-}$ bacterium balnei. Amer. Rev. resp. Dis., 84, 837-844.

Skinner, J. S. (1957). Seal finger. Arch. Derm. 75, 559-561 